\title{
"A SMACK OF IRRELEVANCE" IN INCONSISTENT MATHEMATICS?
}

\author{
Luis Estrada-González and Manuel Eduardo Tapia-Navarro \\ Institute for Philosophical Research, UNAM and Department of Logic, Nicholas \\ Copernicus University in Toruń \\ Graduate Program in Philosophy of Science, UNAM
}

\begin{abstract}
Recently, some proponents and practitioners of inconsistent mathematics have argued that the subject requires a conditional with irrelevant features, i.e. where antecedent and consequent in a valid conditional do not behave as expected in relevance logics - by sharing propositional variables, for example. Here we argue that more fine-grained notions of content and content-sharing are needed to examine the language of (inconsistent) arithmetic and set theory, and that the conditionals needed in inconsistent mathematics are not as irrelevant as it is suggested in the current literature.
\end{abstract}

\section{Introduction}

The question we are interested in here is how much irrelevance is needed in logic for inconsistent mathematics, the program aiming at keeping some naïve principles (which are known to produce contradictions) with a logic weak enough to coexist with them without triviality, but strong enough to recapture a good amount of the most important part (results and proofs) of

Australasian Journal of Logic (18:5) 2021, Article no. 12 
its classical counterpart while obtaining new results. ${ }^{1}$ This is important because according to Weber ([26], [27]) and some of his collaborators (see [12], [2], [13]), a conditional that obeys an irrelevant conditional-introduction rule appears to be indispensable in mathematical proofs and some statements of the form ' $\phi$ entails $\psi$ ' are true even when the entailment makes use of background assumptions other than $\phi$, or does not use $\phi$ at all. In a nutshell, their claim is that an irrelevant conditional is needed for inconsistent mathematics. $^{2}$ However, the concerns about the dates back at least to Meyer's proof that $(m=n) \rightarrow(k=k)$ is valid in his relevant arithmetic $\mathbf{R}^{\#}$ and, in the context of set theory, his remark that one can prove $(x=y) \rightarrow(p \leftrightarrow p)$. These latter conditionals seem objectionable because apparently their respective antecedents and consequents are irrelevant to each other.

In spite of these results, our answer to the opening question is, boldly, that irrelevance has no place in inconsistent mathematics. Less boldly, and following some hints by Meyer himself and Brady, our answer is that the evidence provided thus far only shows that that sort of conditionals are at least weakly relevant in a sense to be made precise below. Much more argument would be needed to say that mathematics requires irrelevant proof methods, or that one can prove irrelevant conditionals in mathematics by following

\footnotetext{
${ }^{1}$ Although inconsistent mathematics is yet to be fully developed, there are already some notable results. See for example the seminal compendium [17], and see [6] for set theory in particular; later developments can be found in [18], [28], [30]. We deliberately omit the approach to mathematics using paraconsistent logic in the style of da Costa and his followers, as such a tradition typically does not work under the assumption that the naïve principles hold good. Rather, they take classical mathematics and classical logic as correct to a great extent, but due to limitative theorems such as Gödel's, according to which contradictions in those theories are possible, they offer means to cope with the contradictions if they happen to arise sometime. See [8] for an overview of the achievements of the (post-)da Costa approach. A more akin approach is the one based on adaptive logics; nonetheless, a useful comparison would require too many detours beyond the scope of this paper. The interested reader might consult [25] for a quick glance at the sort of achievements of the adaptive approach.

${ }^{2}$ But it has to be avoided in some parts of the theory. For example, a conditional satisfying Weakening, that is,
}

Weakening $\Gamma, A \vdash \Gamma, B \rightarrow A$

cannot be used in the formulation of the axiom of Extensionality in inconsistent set theory on pain of triviality, it needs to be another conditional; see [28] and [13, p. 376]. In a slightly different context, Field, Lederman and Øgaard ([11]) have also insisted on the validity of Weakening.

Australasian Journal of Logic (18:5) 2021, Article no. 12 
relevantly acceptable proof methods.

This sort of project need not be based on a "logic first" approach. We might agree pretty much with the practitioners of inconsistent mathematics that mathematical needs are going to drive the shape of the logic, so we are not suggesting a change in the logic(s) they have already "field-tested". Rather, we are questioning the description of what is going in certain aspects of inconsistent mathematics, because it seems odd, whether in classical or inconsistent mathematics, to assert that mathematicians need to prove irrelevant conditionals, especially if that means that antecedent and consequent do not have anything to do with each other. But in order to investigate whether the conditionals have anything to do with each other -i.e. whether they share content or are relevant to each other-, we need more than the Variable Sharing Principle (VSP) or anything in that ballpark, because all those principles were designed for the minimum non-logical bit of zero-order languages. Surprisingly, the VSP made its way as a necessary condition for relevance in higher-order languages too, in spite that there is more content bearers to be analyzed and taken into account in them.

The plan of the paper is as follows. In Section 1 we present the alleged cases of irrelevance in inconsistent mathematics. In Section 2 we outline a theory of weak content that allows for certain conditionals, including the problematic ones in Section 1, to be regarded as at least weakly relevant. In Section 3 we discuss how that proposal might face a problem if set-theoretic reductionism is true.

For simplicity, we will restrict ourselves to arithmetic and set theory. Fitch-style natural deduction would be our preferred format for deploying

and analyzing proofs. In the Appendix you can find a handy presentation of DKQ, which would facilitate the reading of this note for those not familiar with that logic.

\section{The Evidence for Irrelevance}

Famously, Meyer [16, pp. 31 and 36f] proved that $(m=n) \rightarrow(k=k)$ is valid in $\mathbf{R}^{\#}$, i.e. Peano arithmetic formulated with the logic $\mathbf{R}$ as background logic. Here is a sketch of the proof (here and in what follows we omit reiterations for simplicity):

Australasian Journal of Logic (18:5) 2021, Article no. 12 


\begin{tabular}{|c|c|c|}
\hline 1 & $(x-x)=0$ & Elementary algebra \\
\hline 2 & $(0+k)=k$ & Elementary algebra \\
\hline 3 & $m=n$ & Hypothesis \\
\hline 4 & $n=m$ & Symmetry of $=, 3$ \\
\hline 5 & $m=m$ & Transitivity of $=, 3,4$ \\
\hline 6 & $(m-m)=(m-m)$ & Replacement, 5 \\
\hline 7 & $0=0$ & Substitution, 1, 6 \\
\hline 8 & $(0+k)=(0+k)$ & Replacement, 7 \\
\hline 9 & $k=k$ & Substitution, 2, 8 \\
\hline 10 & $(m=n) \rightarrow(k=k)$ & $\mathrm{I} \rightarrow, 3-10$ \\
\hline
\end{tabular}

This means that any equation with a numerical term, regardless of whether it is true or false, entails any true numerical equation.

This result has motivated a number of reactions. Meyer, for example, considered "slightly surprising" and was hesitant between consider it as a fallacy of relevance or rather as an argument for strong relations of mutual relevance between arithmetic propositions [16, p. 31]. Dunn [9, p. 367] is slightly less hesitant: "[t]his is surely somewhat surprising, and smacks of irrelevance." Brady [5, p. 158] also sees a problem of irrelevance:

$m=n \rightarrow m^{\prime}=n^{\prime}$, which has the natural numbers $m$ and $n$ in common, leads to $0=0 \rightarrow 100=100$, where the two numbers involved can be as far apart as you like. Here, the only relevance between ' 0 ' and ' 100 ' is the fact that they are both natural numbers, which is not the same as saying that there is some number in common. So, the relevance is of a different and lesser type than that which would appear to be required by the Relevance Condition [i.e. the Variable-Sharing Principle]. A more straightforwardly irrelevant case, due to Meyer, is $m=n \rightarrow l=l$, which is derivable using transitivity and symmetry, together with the above principle $(\ldots)$. 
Routley's verdict is harsher and declares $\mathbf{R}^{\#}$ irrelevant and filled with "embarrassing difficulties" (see [22, p. 901]). He blames the step from 7 to 8 in the proof above; more generally, he questions the validity of the entailment from $m=n$ to $m+k=n+k$. Like Brady, Routley suggested changing the background logic, to his preferred $\mathbf{D K Q}$, which is stronger than $\mathbf{D} \mathbf{J}^{d} \mathbf{Q}$ (Brady's preferred logic), to avoid the smack of irrelevance. According to Routley, $(m=m) \rightarrow(m+n=m+n)$ should be corrected to $(m=m \wedge n=$ $n) \rightarrow(m+n=m+n)$, just as Factor

$(A \leftrightarrow B) \rightarrow((A \wedge C) \leftrightarrow(B \wedge C))$

should be changed to the weaker alternative:

$((A \leftrightarrow B) \wedge(C \leftrightarrow C)) \rightarrow((A \wedge C) \leftrightarrow(B \wedge C))$

In the case of $(m=m \wedge n=n) \rightarrow(m+n=m+n)$, antecedent and consequent have a number in common; in fact, all numbers in common, as Brady implicitly demanded.

Whatever the merits of Routley's proposal for the case of arithmetic, which are nicely discussed in [19], it is not enough to get rid of any smack of irrelevance in all areas of inconsistent mathematics. Problematic conditionals similar in form to Meyer's have recently been discussed by Weber and Istre in the context of inconsistent set theory. An inconsistent set theory would be a theory based on the following two axioms:

(Unrestricted) Comprehension $\exists y \forall x(x \in y \leftrightarrow \Phi(x, u))$

Extensionality $\quad \forall z(z \in x \leftrightarrow z \in y) \leftrightarrow x=y$

These are known to produce contradictions since Russell (and even Cantor), and there has been a lot of debate on what is the best logic to use for such a theory. Nowadays, the best candidates live around Routley's DKQ. For our purposes, we will stick to that logic; it has a very straightforward natural deduction presentation and it will facilitate exposition and discussion. Thus, in what follows, 'inconsistent set theory' will mean 'DKQ-based naïve set

Australasian Journal of Logic (18:5) 2021, Article no. 12 
theory' unless otherwise stated. ${ }^{3}$

Among the results that are typically expected to be recovered in inconsistent set theory are the certain set inclusions, like the fact that an empty set is a subset of any other set, the equality of sets containing exactly the same members or Mostowski's Collapsing Lemma. These results are important because usually they serve to guarantee that sets have expected and important properties. However, Weber [29] and Istre [12] have argued that proving these requires Weakening. (Field, Lederman and Øgaard [11] make a similar point.)

Let us start with inclusion of an empty set in any other set, defining an empty set as a set $y$ with no elements, that is, such that $\forall x(x \notin y)$. The usual proof that $y$ is a subset of any other set, i.e. $\forall z(y \subseteq z)$, cannot go through because it proceeds via a reductio inadmissible in DKQ, so a different path should be tried to prove $\forall x(x \notin y) \rightarrow \forall z(y \subseteq z)$. In order to prove the consequent, one has to prove $x \in y \rightarrow x \in z$. But this does not go any further. Contraposing, one gets $x \notin z \rightarrow x \notin y$. But the consequent of that conditional already obtains by the hypothesis that $\forall x(x \notin y)$, and nothing but Weakening would do the rest of the job.

Let us move now to the equality of sets. The problem of proving equality from co-extensionality has been in the air for a while. ${ }^{4}$ For example, Weber [27] considered the special case of the equality of two universal sets. Consider the following two sets of a naïve set theory:

$$
\begin{gathered}
U=\{x: x=x\} \\
V=\{x: \exists y(x \in y)\}
\end{gathered}
$$

Both sets have all objects as members, that is, $\forall x(x \in U)$ and $\forall x(x \in V)$. To show $U=V$, would require a proof that $x \in V \rightarrow x \in U$, and Weber thinks that there is no such proof with a relevant conditional because $\exists y(x \in$ $y) \rightarrow(x=x)$ is irrelevant. The easy proof would go as follows:

\footnotetext{
${ }^{3}$ One might wonder whether the problems to be discussed below could be avoided in going straightly to Brady's $\mathbf{D} \mathbf{J}^{d} Q$. The answer is negative. The only difference between $\mathbf{D J}^{d} \mathbf{Q}$ and $\mathbf{D K Q}$ is their treatment of conditionals of the form $A \rightarrow \sim A$ : provided it is a theorem, in $\mathbf{D K Q}$ one can infer $\sim A$ from it, but in $\mathbf{D} \mathbf{J}^{d} \mathbf{Q}$ one cannot even do that. However, no such move is involved in the proofs discussed below. More recently, Brady has advocated an even weaker logic, MC, see [7], but as far as the proofs below are concerned, the situation is very much like as with $\mathbf{D} \mathbf{J}^{d} \mathbf{Q}$ and $\mathbf{D K Q}$.

${ }^{4}$ At least since [3].
}

Australasian Journal of Logic (18:5) 2021, Article no. 12 


\begin{tabular}{|c|c|c|}
\hline 1 & $\forall x(x \in U \leftrightarrow x=x)_{\{\}}$ & Comp. \\
\hline & $\forall x(x \in V \leftrightarrow \exists y(x \in y))_{\{\}}$ & Comp. \\
\hline & $x \in V_{\{1\}}$ & Hypothesis \\
\hline & $\exists y(x \in y)_{\{1\}}$ & $\mathrm{E} \forall x$ and $\mathrm{E} \leftrightarrow, 2,3$ \\
\hline & $x=x_{\{\}}$ & Reflexivity of $=$ \\
\hline & $x \in U_{\{\}}$ & $\mathrm{E} \forall x$ and $\mathrm{E} \leftrightarrow, 1,5$ \\
\hline & $x \in V \rightarrow x \in U_{\{\}}$ & $\mathrm{I} \rightarrow, 2-6$ \\
\hline
\end{tabular}

but it is invalid, because the $\mathrm{I} \rightarrow$ rule cannot be validly applied on step 7 in the logic DKQ. This kind of result means that, in inconsistent set theory, "having the same stuff" is not enough for two sets being equal, but they also must "have the same stuff for similar reasons". This is due to set equality being tied tightly to a conditional formula by Extensionality and by set instantiation being tied so tightly to properties.

An example of having the same stuff for the same reasons is the classical proof of the equality of two finite sets. Consider for example $a=\{0,1,2\}$ and $b=\{0,1,2\}$, which goes as follows:

\begin{tabular}{|c|c|c|}
\hline 1 & $\forall x(x \in a \leftrightarrow(x=0 \vee x=1 \vee x=2))_{\{\}}$ & Comp. \\
\hline 2 & $\forall x(x \in b \leftrightarrow(x=0 \vee x=1 \vee x=2))_{\{\}}$ & Comp. \\
\hline & $0 \in a_{\{1\}}$ & \\
\hline & $0 \in a \leftrightarrow(0=0 \vee 0=1 \vee 0=2)_{\{\}}$ & $\mathrm{E} \forall x x / 0,1$ \\
\hline & $(0=0 \vee 0=1 \vee 0=2)_{\{1\}}$ & $\mathrm{E} \leftrightarrow, 4,3$ \\
\hline & $0 \in b \leftrightarrow(0=0 \vee 0=1 \vee 0=2)_{\{\}}$ & $\mathrm{E} \forall x x / 0,2$ \\
\hline & $0 \in b_{\{1\}}$ & $\mathrm{E} \leftrightarrow, 6,5$ \\
\hline & $0 \in a \rightarrow 0 \in b_{\{\}}$ & $\mathrm{I} \rightarrow, 3-7$ \\
\hline
\end{tabular}

where $a$ and $b$ have the same elements exactly for the same reason, namely Australasian Journal of Logic (18:5) 2021, Article no. 12 
that they instantiate Comprehension in the same way. ${ }^{5}$

As we have said, Mostowski's Collapsing Lemma is another example of a piece of mathematics that is expected to be recovered in inconsistent mathematics. The usual proof is not going to work because it proceeds with a reductio inadmissible in DKQ. The usual proof is long and definitely not as elementary as the previous one, so we will not reproduce it here, but it can be found for example in [14, Ch. 6].

In inconsistent mathematics, that the needed function exists is straightforward because this is a simple instantiation of naïve Comprehension. However, when trying to prove the injectivity of the function, problems arise. Istre [12, p. 56] describes the situation as follows (we have adjusted a little bit the notation):

We would need to derive from our assumption $f(x)=f(y)$ that $\forall z(R z x) \leftrightarrow R z y$ to take advantage of our assumption that $R$ is extensional and thus get $x=y$. Given the definition of $f$, we would need to start by first showing that if some Rax then $f(a) \in f(x)$ as a relevant implication. This move is not possible because it requires $\operatorname{Rax} \rightarrow(\operatorname{Rax} \wedge f(a)=f(a))$ but the second conjunct is irrelevant to $\operatorname{Rax}$. [12, p. 57]

Istre considers other cases where Weakening could be needed. He presents the following hypothetical case:

Mathematical proofs usually take advantage of assumptions which operate as "contextual information", like in the statement "if $x$ and $y$ are ordinals, $x \in O n$ and $y \in O n$, then $x \subseteq y$ or $y \subseteq x$ ". That $x$ and $y$ are ordinals is meant to be used to derive the result. There are a few different paths we could take for such a proof, but the following exhibits the problem.

Assume we attempt to prove to show that $x \subseteq y$ directly after assuming some instances of LEM. This requires proving that $z \in$ $x \rightarrow z \in y$. Since $x$ was arbitrary, we don't know the set property that defined $x$ and so we can't move forward from $z \in x$. We need to use that $x$ is an ordinal to imply some formula $A(z)$, i.e. $x \in O n \vdash A(z)$. Suppose that this property about $z$ is enough to

\footnotetext{
${ }^{5}$ Actually, this example was introduced by Istre [12, p. 32] as a case for the need of Weakening, but the proof is valid in DKQ without relying on Weakening.
}

Australasian Journal of Logic (18:5) 2021, Article no. 12 
get $A(z) \rightarrow z \in y$. It is weakening which would allow us to say that

$$
x \in O n \vdash z \in x \rightarrow A(z)
$$

and thus that

$$
x \in O n \vdash z \in x \rightarrow z \in y \text {. }
$$

Without weakening, we can make no such inference. The use of the assumed information breaks the connection needed for the relevant implication. [12, pp. 34f]

And then he adds on a footnote: "This appears to be another example where weakening plays a unique role in set theory." 6 Here is a sketch of the (hypo-

\begin{tabular}{|c|c|c|}
\hline 1 & $y \in O n_{\{1\}}$ & Hypothesis \\
\hline 2 & $x \in O n_{\{1\}}$ & Hypothesis \\
\hline 3 & $z \in x_{\{2\}}$ & Hypothesis \\
\hline$\vdots$ & $\vdots$ & \\
\hline$n$ & $A(z)_{m}$ & $\ldots, 2$ \\
\hline$\vdots$ & & \\
\hline$m$ & $A(z) \rightarrow z \in y_{k}$ & $\ldots$ \\
\hline$m+1$ & $z \in y_{m, k}$ & $\mathrm{E} \rightarrow, n, m$ \\
\hline$m+2$ & $z \in x \rightarrow z \in y_{\{m, k\}-2}$ & $\mathrm{I} \rightarrow, 3-m+1$ \\
\hline$m+3$ & $x \subseteq y_{\{m, k\}-2}$ & def., $m+2$ \\
\hline$m+4$ & $x \subseteq y \vee y \subseteq x_{\{m, k\}-2}$ & $\mathrm{I} \vee, m+3$ \\
\hline
\end{tabular}
thetical) proof in natural deduction:

${ }^{6}$ These conditionals are discussed again, although in a broader context, in [13].

Australasian Journal of Logic (18:5) 2021, Article no. 12 
where $1,2 \in m$. Note that, according to Istre, step 3 is not used at all beyond serving to start the conditional proof - to prove step $m+1$. Hence, step $m+2$ would be a case of Weakening. The case is too abstract to analyze the proof further. However, it seems to us that step $n$ would be proved not only with step 2 , but that substantial use of 3 would also be made: that $z$ has the property $A$ would hardily be obtained simply from the fact that $x$ is an ordinal, but also from the fact that $z$ is an element of $x .^{7}$ However, let us grant Istre that it is an instance of Weakening, and that step 3 is in fact "deductively inert". That will not affect our main claim in the end of Section 3 , that it is but a special, restricted form of Weakening requiring some sort of relevance which allows proving step $m+2$.

Before moving further, let us recall that for Routley and Brady, Meyer's arithmetical conditionals should be fixed by moving to a weaker logic than $\mathbf{R}$, and that for Weber and Istre, irrelevance is a fact that one should live with in inconsistent mathematics. But not all relevantists share those views. Mortensen thinks, echoing [16, p. 27] that the conditionals above constitute no worry at all: "irrelevance is a property of the theorems and deducibility of logics, not of the nonlogical theorems of arithmetical theories." ([19, 219], italics in the original.) We do not aim to settle the discussion here; we simply take sides with those who think that (ir)relevance can be predicated of mathematical conditionals. ${ }^{8}$

\section{Introducing Weak q-Relevance}

Note the similarity between Istre's and Weber's $\operatorname{Rax} \rightarrow(f(a)=f(a))$ and $\exists y(x \in y) \rightarrow(x=x)$, respectively, and Meyer's $(m=n) \rightarrow(k=k)$. As we saw from Brady's quote in the last section, the first source of the sense of irrelevance arises because one would expect that any valid conditional $A \rightarrow B$ in a relevant theory satisfies the variable-sharing property (VSP):

\footnotetext{
${ }^{7}$ Note that we are not assuming here that if $x$ is an ordinal then $x$ is such and such. Rather, we are saying that it is difficult to get that $z$ is such and such without relying on the fact that $z$ belongs to $x$.

${ }^{8}$ And of any conditionals, we would add. Many references from relevance logicians speaking of relevance and irrelevance of all sorts of conditionals could be mentioned here. To use just one, Mares [15] counts among the "intolerable irrelevancies" the following examples of conditionals: "that any false arithmetical equivalence implies any identity statement"; "cicero $=$ catiline $\rightarrow A$, for every proposition $A$ "; and even "cicero $=$ cicero $\rightarrow$ tully $=$ tully".
}

Australasian Journal of Logic (18:5) 2021, Article no. 12 
VSP $A \rightarrow B$ is a theorem only if there is a propositional variable occurring both in $A$ and $B$.

According to the VSP, the content of a formula is the set of its propositional variables. But $(m=n) \rightarrow(k=k)$ is of the form $A \rightarrow B$, more precisely, $p \rightarrow q$, because antecedent and consequent are atomic, and do not share propositional variables. Mutatis mutandis, something similar can be said of both $\operatorname{Rax} \rightarrow(f(a)=f(a))$ and $\exists y(x \in y) \rightarrow(x=x)$. Hence, irrelevance.

But irrelevance comes in degrees. For example, the following conditionals are not equally irrelevant:

(1) $p \rightarrow(q \rightarrow p)$

(2) $q \rightarrow(p \rightarrow p)$

(3) $(q \rightarrow q) \rightarrow(p \rightarrow p)$

(4) $p \rightarrow(p \rightarrow p)$

(1), an instance of Positive Paradox, is a "dread relevance destroyer" 9 and cannot be added to a logic in the relevance family without collapsing it in classical logic, and the same happens with (2). Prior famously held that paradoxes of strict implication might in fact be proofs that "necessary and impossible propositions as such have a definite inner connexion with all propositions whatever" [20, p. 196,; italics in the original]. This cannot be unrestrictedly the case in relevance logics, for (2) implies a collapse equal to (1). But the "definite inner connection" between any two necessary implications, as in (3), can be added to relevance logics without collapse, producing the $\mathbf{I}$ logics, for example. ${ }^{10}$ Even more, the definite inner connection can be assumed for a necessary proposition and any contingent proposition occurring in it, as in (4), which has lead to RM and other "Minglish" logics. ${ }^{11}$

The fact that (1) is so irrelevant in spite of meeting the VSP suggests that another condition for relevance, necessary at least, and stronger than VSP, is operating in $\mathbf{R}$. Such a condition, introduced in [1, Ch. IV, §22.1.3], is the

\footnotetext{
${ }^{9}$ As Dunn [9, p. 355] colorfully calls it.

${ }^{10}$ See [21] for a more detailed examination of these logics.

${ }^{11} \mathbf{R M}$ has been widely studied by relevance logicians. For the basics, see [1, §29.4].
} 
No-loose pieces property (NLPP) if $A \rightarrow B$ is a purely implicative theorem, every variable occurs in $A$ if and only if it occurs in $B$.

Also, not as important but also highly desirable is the

Ackermann property (AP) there is no theorem of the form $A \rightarrow(B \rightarrow$ $C)$ where $A$ contains no ' $\rightarrow$ '.

The following table systematizes the features of the conditionals above:

\begin{tabular}{|c|l|l|l|}
\hline & $\mathrm{NLPP}$ & $\mathrm{VSP}$ & $\mathrm{AD}$ \\
\hline$p \rightarrow(q \rightarrow p)$ & $\times$ & $\checkmark$ & $\times$ \\
\hline$q \rightarrow(p \rightarrow p)$ & $\times$ & $\times$ & $\times$ \\
\hline$(q \rightarrow q) \rightarrow(p \rightarrow p)$ & $\times$ & $\times$ & $\checkmark$ \\
\hline$p \rightarrow(p \rightarrow p)$ & $\checkmark$ & $\checkmark$ & $\times$ \\
\hline
\end{tabular}

It shows clearly that (1) and (2) are totally inadmissible. (3) is bad but there is some redemption for it if one is liberal enough. (4) is bad but it is the least bad of the four given these desiderata. ${ }^{12}$

That (3) can be added to $\mathbf{R}$ without collapsing it in classical logic means that VSP and NLPP can be relaxed in a way that (1) and (2) are still out of the relevance realm. In fact, in the literature there are other, more liberal, necessary conditions for relevance, reflecting the differences between these conditionals. ${ }^{13}$ A relaxed condition that can make room for (3) is ultra-weak relevance $(\mathrm{UwR})$ :

UwR $A \rightarrow B$ is valid only if $A$ and $B$ share a propositional variable or a connective.

According to this, the content of a formula is the set of its propositional variables or connectives. As we have said, this allows going beyond $\mathbf{R}$ to the I systems, where schemes like (3) above, $(B \rightarrow B) \rightarrow(A \rightarrow A)$, are valid.

\footnotetext{
${ }^{12}$ One could wonder why (3) is better than (1) if both meet just one of the desiderata. The short story is that (3) scores better by relevantists' lights because in (3) not only an entailment is entailed by another entailment, unlike in (1), but also both antecedent and consequent in (3) share modal status; that a necessary entailment entails another necessary entailment is less objectionable than having an atomic, contingent proposition entailing a necessary entailment.

${ }^{13}$ For an overview of some of those conditions, see [23].
}

Australasian Journal of Logic (18:5) 2021, Article no. 12 
Although UwR does not help for our present purposes- $(m=n) \rightarrow(k=$ $k$ ) still has the propositional structure $p \rightarrow q-$, it does give a good hint on what is needed, namely, considering the more fine-grained structure of propositions so that the shareable content is not exhausted with propositional variables or the connectives connecting them. VSP and UwR as stated cover just zero-order logic and would render invalid conditionals like $F a \rightarrow \exists x F x$ and $\forall x F x \rightarrow F a$, which are prima facie not relevantly objectionable. Thus, let us define weak q-content as follows:

Weak q-content The weak q-content of $A$ is the set of its terms and (relevant) predicates.

This allows us to define weak q-relevance as follows:

Weak q-relevance $A \rightarrow B$ is valid only if $A$ and $B$ share weak q-content, where sharing is understood as set intersection. ${ }^{14}$

Clearly, $(m=n) \rightarrow(k=k)$ is at least weakly q-relevant; moreover, $(m=n)$ is used in the proof of $(k=k)$, the latter is not simply introduced as a logical truth. Note that Brady and perhaps also Routley demand something stronger, namely that the q-content is just the set of terms and that antecedent and consequent in a valid conditional have the same q-content.

Let us consider now the problematic set-theoretic conditionals of Section 1 :

(W1) $x \notin z \rightarrow x \notin y$

(W2) $0 \in b \rightarrow 0 \in a$

(W3) $\exists y(x \in y) \rightarrow(x=x)$

(W4) $\operatorname{Rax} \rightarrow(f(a)=f(a))$

Note that, in each case, the antecedent is not that arbitrary with respect to the consequent: they share at least terms, and some of them share also (relevant) predicates, so they all are at least weakly q-relevant to each other.

Now, as in the case of the irrelevant formulas (1)-(4) above, there is a way in which one can systematize the dissatisfaction with (W1)-(W4):

\footnotetext{
${ }^{14}$ The reader can decide what the ' $\mathrm{q}$ ' means here. It can stand for 'quantificational structure', 'quasi-relevance' or whatnot.
}

Australasian Journal of Logic (18:5) 2021, Article no. 12 


\begin{tabular}{|c|l|l|l|}
\hline & $\begin{array}{l}\text { Antecedent } \\
\text { and consequent } \\
\text { share terms }\end{array}$ & $\begin{array}{l}\text { Antecedent } \\
\text { and consequent } \\
\text { share relevant } \\
\text { properties }\end{array}$ & $\begin{array}{l}\text { Antecedent and } \\
\text { consequent have } \\
\text { the same modal } \\
\text { status }\end{array}$ \\
\hline$x \notin z \rightarrow x \notin y$ & $\checkmark$ & $\checkmark$ & $\checkmark$ \\
\hline $0 \in b \rightarrow 0 \in a$ & $\checkmark$ & $\checkmark$ & $\checkmark$ \\
\hline$\exists y(x \in y) \rightarrow(x=x)$ & $\checkmark$ & $\times$ & $\times$ \\
\hline $\operatorname{Rax} \rightarrow(f(a)=f(a))$ & $\checkmark$ & $\times$ & $\times$ \\
\hline
\end{tabular}

All of them look suspicious because they fail VSP. Nonetheless, a more detailed examination of the atomic propositions show that in all cases there is more fined-grained content shared between the components of these conditionals. Also, the dissatisfaction with (W3) and (W4) is greater because they score really low, although still they keep the minimum of relevance which prevents the need for unrestricted Weakening in full force. In that sense, (W3) and (W4) resemble Mingle: a necessary consequent, that can be proved on its own, is implied by a contingent proposition. Nonetheless, there is some redemption to Mingle because that contingent proposition occurs in the necessary one, unlike the case of $q \rightarrow(p \rightarrow p)$. The situation is pretty much the same with (W3) and (W4).

The $\mathrm{W}$ conditionals are unlike $(m=n) \rightarrow(k=k)$ in that their antecedents are not used at all in proving the consequent; in the proofs of those conditionals, the antecedents are mere hypotheses to start the conditional proof. However, it is easy to see then that the rule needed in these examples of "irrelevant" deductions is not Weakening tout court but

Restricted Weakening If $\Gamma \vdash A$ then $\Gamma \vdash B \rightarrow A$ provided that $A$ and $B$ share at least weak q-content.

To finalize this section, let us note that there is a "smack of irrelevance" already in the axioms that would be nice to cover with our suggestion. ${ }^{15}$ As for Extensionality, a universally quantified biconditional is connected to an identity with a biconditional. In this case, antecedents and consequents in both conditionals share terms, $x$ and $y$. For Comprehension, we have a biconditional between a membership statement and some arbitrary other statement $\Phi(x, u)$ that could just be e.g. some proposition $p$. In the original formulation, the membership statement $x \in y$ and the statement $\Phi(x, u)$

\footnotetext{
${ }^{15}$ Thanks to Zach Weber for pressing us to discuss this point.
}

Australasian Journal of Logic (18:5) 2021, Article no. 12 
share terms, which is lost if the latter is re-written as ' $p$ ', as Meyer and others found. Here is where the "syntactic" approaches to content-sharing find their limit, because they are too sensitive to language manipulation. Nonetheless, we hope to have showed that the attempt of showing that the smack of irrelevance in axioms is not real, full irrelevance, is not hopeless.

\section{4 (Restricted) Weakening and Set-Theoretic Reductionism}

But happy endings are not the norm in inconsistent mathematics. Suppose material set-theoretic reductionism, the idea that every mathematical statement is equivalent to one about sets and membership between them, is true. Then any two mathematical statements are at least weakly q-relevant to each other as the membership relation is shared among any two mathematical statements. If that is so, the proverbial conjecture about Banach spaces $(B)$ is relevant both to the completeness of first-order calculus $(C)$ and to Fermat's Last Theorem $(F)$. But this seems to go against the original relevantist desiderata. ${ }^{16}$

One can try to get rid of this worry by pointing out that even if $B$ is relevant to $C$, in the sense that they share (weak q-)content, that is not enough for the validity of $B \rightarrow C$ : at least a proof of $C$ that uses $B$ is needed. (And, mutatis mutandis, the same goes for $B \rightarrow F$.) However, under set-theoretic reductionism, Restricted Weakening collapses into Weakening simpliciter: any conditional $A \rightarrow B$, with $B$ a theorem, is valid, because the restriction is automatically satisfied given that set-theoretic membership would be present in any formula.

We will not try to find a way out of this conundrum here, but there are many options available. Nonetheless, we are not to develop them fully nor evaluate them properly here; that would require a separate work. First, there is the option of rejecting the idea that membership is a relevant property. This would be problematic because membership satisfies the best working definition of relevant property, that of Dunn [9, p. 357]. A less radical idea would be exploring membership as a relevant property but only of the con-

\footnotetext{
${ }^{16}$ The problem will arise for any signature with only one ( $n$-ary) predicate. For definiteness, and for its central role in foundational discussions, we stick to the particular case of set theory.
}

Australasian Journal of Logic (18:5) 2021, Article no. 12 
taining set, not of the member, that is, giving radically different logical treatments to the properties "having $x$ as a member" and "being a member of $y$ ", as suggested by Dunn himself in $[10$, p. 90f $]$ ). Having the elements that it has could be a relevant property of a set, but belonging to this or that set in general could be not relevant for the members. Modifying Dunn's example a little bit, having $\varnothing$ and France as members is a relevant property of $\{\varnothing$, France $\}$, but belonging to $\{\varnothing$, France $\}$ does not seem a relevant property of either $\varnothing$ or France.

Finally, there is the option of considering set-theoretic reductionism false in the case of inconsistent mathematics. There are some arguments to the effect that it is very implausible for set theories based on logics in the vicinity of LP; see for example [24]. Set theories based on logics at least as strong as DKQ seem to contain too much "noise", that is, extraneous inconsistent facts, such as $(r \in r \wedge r \notin r) \rightarrow \sim(r \in r \rightarrow r \in r)$. Moreover, sets like Routley's — the set $\mathcal{Z}$ of all sets not belonging to $\mathcal{Z}$; see [22] — appear to generate an enormous amount of inconsistency at all levels; for example, everything belongs and does not belong to it and, moreover, it might make all arguments invalid (even if some are valid too). Up to now, it is unclear whether these features of an inconsistent set theory would have an impact on the reconstruction of other mathematical theories within it.

\section{Conclusions}

In this paper, we have proposed the notions of weak q-content and weak qrelevance to defend the thesis that certain conditionals in inconsistent mathematics are not completely irrelevant, in the sense that their antecedents and consequents do not share content. This would alleviate, at least partially, some concerns raised by certain theorems proved by Meyer in relevant arithmetic, like $(m=n) \rightarrow(k=k)$. Those notions are also useful in showing that some apparent uses of unrestricted Weakening in mathematics are actually instances of a restricted version of such a rule. It is important to emphasize that we are not defending the idea that (in general) this shows that unrestricted Weakening is not necessary for inconsistent mathematics, but rather that the evidence provided so far for the contrary is insufficient. We believe that the most important conclusion to be drawn from this work is that a notion of relevance that encompass a fine-grained notion of logical content, besides just propositional content, is most necessary. We believe

Australasian Journal of Logic (18:5) 2021, Article no. 12 
that an adequate notion of logical content must include an account of the content involved in terms or predicates, which seems important in cases like those exposed by Meyer, Routley, Brady, Weber, Istre and others. It is our hope that this note advanced the discussion in that direction.

\section{Appendix: DKQ}

Here we present axiomatic and natural deduction versions of DKQ following $[4]$.

\section{Hilbert-Style DKQ}

\section{Axiom schemas}

1. $A \rightarrow A$

2. $(A \wedge B) \rightarrow A$

3. $(A \wedge B) \rightarrow B$

4. $((A \rightarrow B) \wedge(A \rightarrow C)) \rightarrow(A \rightarrow(B \wedge C))$

5. $A \rightarrow(A \vee B)$

6. $B \rightarrow(A \vee B)$

7. $((A \rightarrow C) \wedge(B \rightarrow C)) \rightarrow((A \vee B) \rightarrow C)$

8. $(A \wedge(B \vee C)) \rightarrow((A \wedge B) \vee(A \wedge C))$

9. $\sim \sim A \rightarrow A$

10. $(A \rightarrow \sim B) \rightarrow(B \rightarrow \sim A)$

11. $((A \rightarrow B) \wedge(B \rightarrow C)) \rightarrow(A \rightarrow C)$

12. $A \vee \sim A$

13. $\forall x A \rightarrow A[y / x], y$ free for $x$ in $A$

14. $\forall x(A \rightarrow B) \rightarrow(A \rightarrow \forall x B), x$ not free in $A$ 
15. $\forall x(A \vee B) \rightarrow(A \vee \forall x B), x$ not free in $A$

16. $A[y / x] \rightarrow \exists x A, y$ free for $x$ in $A$

17. $\forall x(A \rightarrow B) \rightarrow(\exists x A \rightarrow B), x$ not free in $B$

18. $(A \wedge \exists x B) \rightarrow \exists x(A \wedge B), x$ not free in $A$

\section{Rules}

R1. $A, A \rightarrow B \vdash B$

R2. $A, B \vdash A \wedge B$

R3. $A \rightarrow B, C \rightarrow D \vdash((B \rightarrow C) \rightarrow(A \rightarrow D))$

R4. $A \vdash \forall x A$

R5. $x=y \vdash A(x) \rightarrow A(y)$

\section{Natural Deduction System for DKQ}

Hyp. A formula $A$ may be introduced as the hypothesis of a new subproof, with a subscript $\{\mathrm{k}\}$, where $\mathrm{k}$ is the rank of the new subproof

Reit. $A_{a}$ may be reiterated to the right into any subproof containing $A_{a}$ retaining the index set $a$

$\rightarrow$ I From a proof of $B_{a}$ on the hypothesis $A_{\{k\}}$, to infer $A \rightarrow B_{a-\{k\}}$ in the next outer subproof, provided $k \in a$

$\rightarrow$ E From $A_{a}$ and $A \rightarrow B_{b}$ to infer $B_{a \cup b}$, provided the DKQ-restriction holds

$\sim$ I From $A \rightarrow \sim A_{\varnothing}$ to infer $\sim A_{\varnothing}$, provided the DKQ-restriction holds

$\sim$ E From $\sim B_{a}$ and $A \rightarrow B_{b}$ to infer $\sim A_{a \cup b}$, provided the DKQ-restriction holds

$\sim \sim \mathbf{I}$ From $A_{a}$ to infer $\sim A_{a}$

$\sim \sim \mathbf{E}$ From $\sim A_{a}$ to infer $A_{a}$ 
$\wedge \mathbf{I}$ From $A_{a}$ and $B_{a}$ to infer $A \wedge B_{a}$

$\wedge \mathbf{E}$ From $A \wedge B_{a}$ to infer $A_{a}$ From $A \wedge B_{a}$ to infer $B_{a}$

$\vee \mathbf{I}$ From $A_{a}$ to infer $A \vee B_{a}$ From $B_{a}$ to infer $A \vee B_{a}$

$\vee \mathbf{E}$ From $A \vee B_{a}, A \rightarrow C_{b}$ and $B \rightarrow C_{b}$ to infer $C_{a \cup b}$, provided the DKQrestriction holds

$\wedge \vee$ From $A \wedge(B \vee C)$ to infer $(A \wedge B) \vee(A \wedge C)$

$\forall x$ I From $A_{a}$ to infer $\forall x A_{a}$ provided $\mathrm{x}$ is not free in any hypothesis $H_{\{k\}}$ with $k \in a$

$\forall x \mathbf{E}$ From $\forall x A_{a}$ to infer $A[y / x]_{a}$ where $y$ is free for $x$ in $A$

$\forall x \vee$ From $\forall x(A \vee B)_{a}$ to infer $A \vee \forall x B_{a}$, where $x$ is not free in $A$

$\exists x \mathbf{I}$ From $A[y / x]_{a}$ to infer $\exists x A_{a}$ where $y$ is free for $x$ in $A$

$\exists x \mathbf{E}$ From $\exists x A_{a}$ and $\forall x(A \rightarrow B)_{b}$ to infer $B_{a \cup b}$ where $x$ is not free in $B$ and provided DKQ-restriction holds

$\exists x \wedge$ From $A \wedge \exists x B_{a}$ to infer $\exists x(A \wedge B)_{a}$ where $x$ is not free in $A$

DKQ-Restriction If $b \neq \varnothing$ then (i) $a \neq \varnothing$, (ii) $\max (b)<\max (a)$ and (iii) $a-\{\max (a)\}=a$ or $\varnothing$

Finally, $A \leftrightarrow B$ is defined as $(A \rightarrow B) \wedge(B \rightarrow A)$ as expected, so the rules of introduction and elimination for $\leftrightarrow$ are much the same rules for $\wedge$, with the DKQ-restriction in the elimination.

\section{Acknowledgments}

This work was supported by the PAPIIT project IN403719. Previous versions were presented at the First Bilateral Workshop UniCa-UNAM and at the Logic Colloquium 2019. We would like to thank Zach Weber, Francesco Paoli, Axel Barceló, Michèle Friend, Andrew J. Tedder, Francisco MartínezAviña, Fernando Cano-Jorge and Erik Istre for their useful comments, as well as Charlie Donahue for his encouragement.

Australasian Journal of Logic (18:5) 2021, Article no. 12 


\section{References}

[1] A. R. Anderson and N. D. Belnap. Entailment: The Logic of Relevance and Necessity, Vol. I. Princeton University Press, Princeton, 1975.

[2] G. Badía and Z. Weber. A substructural logic for inconsistent mathematics. In A. Reiger and G. Young, editors, Dialetheism and its Applications, pages 155-176. Springer, Cham, 2019.

[3] R. T. Brady. The consistency of the axioms of abstraction and extensionality in a three valued logic. Notre Dame Journal of Formal Logic, 12:447-453, 1971.

[4] R. T. Brady. Natural deduction systems for some quantified relevant logics. Logique Et Analyse, 27(8):355-377, 1984.

[5] R. T. Brady. Relevant implication and the case for a weaker logic. Journal of Philosophical Logic, 25:151-183, 1996.

[6] R. T. Brady. Universal Logic. CSLI Publications, CSLI Notes Lectures Notes, 2006.

[7] R. T. Brady. On the law of excluded middle. In Z. Weber, editor, Ultralogic as Universal? The Sylvan Jungle, Volume 4, pages 161-184. Springer, Cham, 2019.

[8] W. Carnielli and M. E. Coniglio. Paraconsistent Logic: Consistency, Contradiction and Negation. Logic, Epistemology, and the Unity of Science. Springer, 2016.

[9] J. M. Dunn. Relevant predication 1: The formal theory. Journal of Philosophical Logic, 16(4):347-381, 1987.

[10] J. M. Dunn. Relevant predication 3: Essential properties. In J. M. Dunn and A. Gupta, editors, Truth or Consequences. Essays in Honor of Nuel Belnap. Kluwer Academic Publishers, 1990.

[11] H. Field, H. Lederman, and T. F. Øgaard. Prospects for a naive theory of classes. Notre Dame Journal of Formal Logic, 58(4):461-506, 2017.

[12] E. Istre. Normalized Naive Set Theory. University of Canterbury, 2017.

[13] E. Istre and M. McKubre-Jordens. The difficulties in using weak relevant logics for naive set theory. In C. Başkent and T. M. Ferguson, editors, Graham Priest on Dialetheism and Paraconsistency, pages 365381. Springer Verlag, 2019.

[14] T. Jech. Set Theory. Springer, 3rd edition, 2002.

[15] E. D. Mares. Relevant Logic: A Philosophical Interpretation. Cambridge University Press, 2004.

[16] R. K. Meyer. The consistency of arithmetic. 1975.

Australasian Journal of Logic (18:5) 2021, Article no. 12 
[17] C. Mortensen. Inconsistent Mathematics. Kluwer Mathematics and Its Applications Series. Kluwer Academic Publishers, Dordrecht, 1995.

[18] C. Mortensen. Inconsistent Geometry. Number 27 in Studies in Logic. College Publications, 2010.

[19] C. Mortensen. Implication principles in Routley Arithmetic. In Z. Weber, editor, Ultralogic as Universal? The Sylvan Jungle, Volume 4, pages 185-194. Springer, Cham, 2019.

[20] A. N. Prior. Formal Logic. Clarendon Press, Oxford, 1955.

[21] R. Routley. A semantical analysis of implication system $I$ and of the first degree entailment. Mathematische Annalen, 196(1):58-64, 1972.

[22] R. Routley. Ultralogic as universal? In Exploring Meinong's Jungle and Beyond, pages 893-959. Philosophy Department, RSSS, Australian National University, 1977. New edition by Z. Weber, editor, Ultralogic as Universal? The Sylvan Jungle, Volume 4, Springer, Cham, 2019.

[23] R. Sylvan. An orientational survey of sociative logics. In Bystanders' Guide to Sociative Logics. Australian National University, Canberra, 1989. Reprint with minor modifications in Sociative Logics and Their Applications: Essays by the Late Richard Sylvan, D. Hyde and G. Priest, eds., Ashgate, pp. 29-51, 2000.

[24] N. Thomas. Expressive limitations of naive set theory in LP and minimally inconsistent LP. The Review of Symbolic Logic, 7(2):341-350, 2014.

[25] P. Verdée. Strong, universal and provably non-trivial set theory by means of adaptive logic. Logic Journal of the IGPL, 21(1):108-125, 2013.

[26] Z. Weber. Inconsistent mathematics. In J. Fieser and B. Dowden, editors, Internet Encyclopedia of Philosophy. 2009.

[27] Z. Weber. Extensionality and restriction in naive set theory. Studia Logica, 94(1):87-104, 2010.

[28] Z. Weber. Transfinite numbers in paraconsistent set theory. The Review of Symbolic Logic, 3(1):71-92, 2010.

[29] Z. Weber. Transfinite cardinals in paraconsistent set theory. The Review of Symbolic Logic, 5(2):269-293, 2012.

[30] Z. Weber and M. McKubre-Jordens. Real analysis in paraconsistent logic. Journal of Philosophical Logic, 41(5):901-922, 2012.

Australasian Journal of Logic (18:5) 2021, Article no. 12 